\title{
A Pilot Study for Early Detection of Oral Premalignant Diseases Using Oral Cytology and Raman Micro-Spectroscopy: Assessment of Confounding Factors
}

\author{
Isha Behl \\ Technological University Dublin, isha.behl@tudublin.ie \\ Genecy Calado \\ Technological University Dublin \\ Alison Malkin \\ Technological University Dublin, alison.malkin@tudublin.ie
}

See next page for additional authors

Follow this and additional works at: https://arrow.tudublin.ie/radart

Part of the Dentistry Commons

\begin{abstract}
Recommended Citation
Behl, Isha; Calado, Genecy; Malkin, Alison; Flint, Stephen; Galvin, Sheila; Healy, Claire; Pimentel, Marina Leite; Byrne, Hugh; and Lyng, Fiona, "A Pilot Study for Early Detection of Oral Premalignant Diseases Using Oral Cytology and Raman Micro-Spectroscopy: Assessment of Confounding Factors" (2020). Articles. 79. https://arrow.tudublin.ie/radart/79
\end{abstract}

This Article is brought to you for free and open access by the Radiation and Environmental Science Centre at ARROW@TU Dublin. It has been accepted for inclusion in Articles by an authorized administrator of ARROW@TU

Dublin. For more information, please contact

arrow.admin@tudublin.ie, aisling.coyne@tudublin.ie, gerard.connolly@tudublin.ie.

Funder: Science Foundation Ireland

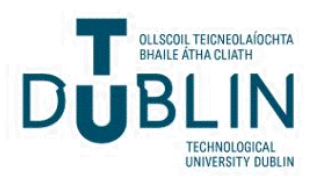


Authors

Isha Behl, Genecy Calado, Alison Malkin, Stephen Flint, Sheila Galvin, Claire Healy, Marina Leite Pimentel, Hugh Byrne, and Fiona Lyng

This article is available at ARROW@TU Dublin: https://arrow.tudublin.ie/radart/79 


\title{
A pilot study for early detection of oral premalignant diseases using oral cytology and Raman micro-spectroscopy: Assessment of confounding factors
}

\author{
Isha Behl $^{1,2 *}$ (1) | Genecy Calado ${ }^{1,2}$ | Alison Malkin ${ }^{3}$ | Stephen Flint $^{4}$ | \\ Sheila Galvin $^{4}$ | Claire M. Healy ${ }^{4}$ | Marina Leite Pimentel ${ }^{5}$ | \\ Hugh J. Byrne ${ }^{6}$ (i) | Fiona M. Lyng ${ }^{1,2}$ (i)
}

${ }^{1}$ Centre for Radiation and Environmental Science, FOCAS Research Institute, Technological University Dublin. City Campus, Dublin, Ireland

${ }^{2}$ School of Physics \& Clinical \& Optometric Sciences, Technological University Dublin, City Campus, Dublin, Ireland

${ }^{3}$ School of Biological and Health Sciences, Technological University Dublin, City Campus, Dublin, Ireland

${ }^{4}$ Oral Medicine Unit, Dublin Dental University Hospital, Trinity College Dublin, Dublin, Ireland

${ }^{5}$ Division of Restorative Dentistry and Periodontology, Dublin Dental University Hospital, Trinity College Dublin, Dublin, Ireland

${ }^{6}$ FOCAS Research Institute, Technological University Dublin, City Campus, Dublin, Ireland

\section{*Correspondence}

Dr Isha Behl, Centre for Radiation and Environmental Science, FOCAS Research Institute, Technological University Dublin, City Campus, Dublin, Ireland. Email: behlisha86@gmail.com

\section{Funding information}

Fiosraigh Dean of Graduate Studies Award; Science Foundation Ireland, Grant/Award Number: 12/IP/1494; Technological University Dublin

\begin{abstract}
This study demonstrates the efficacy of Raman micro-spectroscopy of oral cytological samples for differentiating dysplastic, potentially malignant lesions from those of normal, healthy donors. Cells were collected using brush biopsy from healthy donors $(\mathrm{n}=20)$ and patients attending a

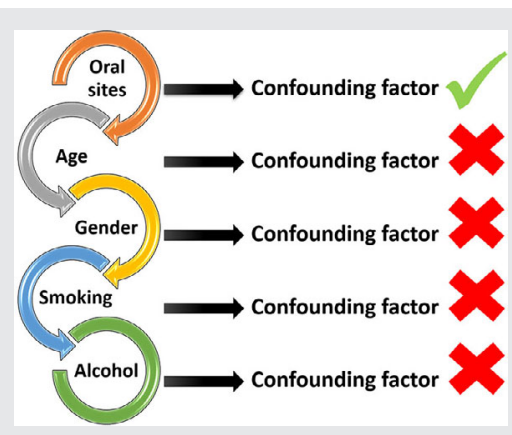
Dysplasia Clinic $(n=20)$. Donors were sampled at four different sites (buccal mucosa, tongue, alveolus, gingiva), to ensure matched normal sites for all lesions, while patient samples were taken from clinically evident, histologically verified dysplastic lesions. Spectra were acquired from the nucleus and cytoplasm of individual cells of all samples and subjected to partial least squares-discriminant analysis. Discriminative sensitivities of $94 \%$ and $86 \%$ and specificity of $85 \%$ were achieved for the cytoplasm and nucleus, respectively, largely based on lipidic contributions of dysplastic cells. Alveolar/gingival samples were differentiated from tongue/buccal samples, indicating that anatomical site is potentially a confounding factor, while age, gender, smoking and alcohol consumption were confirmed not to be.
\end{abstract}

\section{KE Y W O R D S}

oral brush biopsy cytological samples, oral potentially malignant lesions, partial least squaresdiscriminant analysis, Raman micro-spectroscopy, sensitivity and specificity

\footnotetext{
Abbreviations: ANOVA, analysis of variance; CIS, carcinoma in-situ; LOPOCV, leave one patient out cross validation; LV, latent variable; OSCC, oral squamous cell carcinoma; PLS-DA, partial least squaresdiscriminant analysis; PCA, principal components analysis.
}

\section{1 | INTRODUCTION}

Carcinoma of the lip and oral cavity ranks as the 18th most common cancer in the world and the 8th most 
frequent cancer in males [1]. It has an overall 5-year survival rate of approximately $50 \%$, due mostly to late stage diagnosis [2]. Oral squamous cell carcinomas (OSCCs) can either arise de novo or from potentially malignant lesions, typically either from a leukoplakia (white patch), erythroplakia (red patch) or erythroleukoplakia (mixed red and white patch) [3]. Such lesions, if found to be dysplastic, have high probability of developing into malignant lesions, and are therefore clinically referred to as potentially malignant lesions. The low rate of early diagnosis of OSCC can be partially attributed to the late recognition of such potentially malignant lesions, prior to malignant transformation $[4,5]$.

The gold standard for the diagnosis of potentially malignant lesions is biopsy and histopathological examination, in order to determine the presence and degree of dysplasia, based on the cell morphology and tissue architecture. Furthermore, the degree of dysplasia of the biopsy is currently regarded as the best predictor of malignant transformation of potentially malignant lesions [2]. Patients with oral leukoplakia, erythroplakia and erythroleukoplakia need to be followed up long term and often require repeated biopsies. There is, therefore, an unmet clinical need for new technology to reduce the need for multiple invasive tissue biopsies. As an alternative, brush-biopsy cytology is a simple, minimally invasive procedure, which is well accepted by patients $[6,7]$.

The oral mucosa is divided into three basic types of epithelium, depending on the site and function: masticatory, lining and specialised epithelium [8,9]. Gingiva and hard palate are the sites of the oral cavity which are subject to mechanical forces associated with mastication. These sites are covered by keratinising epithelium resembling that of the epidermis covering the skin. The dorsal surface of the tongue is a specialised epithelium, which can be represented as keratinised and non-keratinised epithelium $[8,9]$.

The brush biopsy samples are representative of the stratified epithelial layer of the oral mucosa which is comprised of three layers of epithelium; basal, intermediate and superficial layer. The basal layer is the only layer of epithelium which has dividing cells, from where cells mature and exfoliate on the surface of the epithelium (superficial layer) [10]. However, it is critical that the profile of the harvested sample contains the cells of interest. In the case of mild dysplasia, histopathologically abnormal cells are present in the basal and parabasal cell layers of the oral epithelium. As these abnormal cells are deep inside the tissue, it is difficult to harvest them in a typical cytopathological sample [11], which would mostly comprise of morphologically normal cells from the surface, leading to false negative results. False-positive and falsenegative results in oral cancer diagnosis using oral cytology are often attributed to the fact that the cellular sample was not collected from the full thickness of the epithelium [11].

While assessing the potentially malignant lesion samples, cytomorphological features, such as the nuclear area (NA), cytoplasmic area (CA), and nucleus-to-cytoplasmic area ratio (NA/CA) are the major features that are considered in the conventional cytopathological evaluation of exfoliated cells. It is well-known, however, that exfoliated cells from the healthy donors can differ morphologically, depending on age, gender, smoking and alcohol consumption habits $[2,3,12,13]$. It has previously been reported that exfoliated cells of different genders exhibit morphological differences due to hormonal changes in females throughout their lifetime, from puberty to menopausal age [13]. According to an earlier report, a significant age-related variation can be observed morphologically in terms of NA, CA, NA/CA [13]. This age-related variation can be ascribed to cellular senescence [14]. The renewal capacity of the basal cell layer of normal epithelium reduces with age, resulting in the accumulation of senescent cells. The cells which remain for a longer duration in the oral cavity succumb to the effect of various local environmental factors $[8,13]$.

It has also been reported that the keratinisation level and nucleolar activity increases in clinically normal mucosa of smokers [15]. Moreover, a greater percentage of enucleated superficial cells and presence of micronuclei can be seen in the normal exfoliated cells of smokers [15-17]. In addition, increase in nuclear size, decrease in basal cell size and increase in number of mitotic figures with pyknosis, karyorrhexis and karyolysis have been reported previously due to alcohol consumption $[18,19]$.

Rather than relying on the morphological appearance, optical spectroscopic methods such as infrared (IR) absorption and Raman scattering are sensitive to the molecular composition of cells and tissue. Both techniques provide information on the vibrational frequencies of different macro-molecules (lipids, proteins, nucleic acid and carbohydrates) and are ideally poised to provide alternative methods for diagnosis of oral dysplasia [4]. Combining information from molecular profiles with cytological techniques may overcome the limitations of cytology [11]. Previously, FTIR and Raman spectroscopy has shown potential to detect oral cancer and precancerous changes using exfoliated cells [20-25].

In the present study, using the standardised protocol of Behl et al [26] for Raman micro-spectroscopic analysis of oral cytological samples, classification models were constructed using partial least squares-discriminant analysis (PLS-DA) with leave one patient out cross validation (LOPOCV) to differentiate the Raman spectra of healthy 
donors from premalignant patient samples. Concurrently, it is also vital to test whether potential confounding factors of oral sampling site, gender, age, smoking and alcohol consumption can compromise the sensitivity and specificity of the Raman micro-spectroscopic technique. The rationale and the clinical aim for the study are to develop a test that could reduce the need for multiple biopsies for patients with potentially malignant lesions. The implication of such potential confounding factors for early oral cancer detection based on Raman microspectroscopy of brush biopsy samples were therefore assessed.

\section{2 | MATERIALS AND METHODS}

\section{1 | Sample collection and processing}

For this pilot study, oral brush biopsy samples were collected from healthy donors $(n=20)$ in the FOCAS Research Institute (in-house), Technological University Dublin, using endocervical brushes (Aquilant Scientific, Dublin, Ireland), and from patients $(\mathrm{n}=20)$ with dysplastic oral lesions attending the Oral Mucosa Dysplasia Clinic of the Dublin Dental University Hospital. Oral brush biopsy cytological samples were collected from the identified dysplastic lesion in the patient group and from four different sites (buccal mucosa, ventral surface of the tongue, alveolus mucosa and gingiva) in healthy donors to ensure matched normal sites for all lesions. The healthy donors were aged between 25 and 60 years and patients between 40 and 90 years. The percentage of male, female, was approximately matched $(\sim 55 \%, 45 \%)$. Ethical approval was obtained from the St James' Hospital/Tallaght Hospital Joint Research Ethics Committee (REC ref: 2013/23/05) to collect the patient samples, and from the Dublin Institute of Technology (now TU Dublin) Research Ethics Committee (REC ref: 15/104) for the collection of healthy donor samples. Written informed consent was obtained from each donor and the study was conducted in accordance with ethical principles founded in the Declaration of Helsinki.

The healthy donors were screened before sampling by a trained dentist who also supervised the sample collection. The oral mucosa of the healthy donors was not biopsied. Patients were sampled by an oral medicine consultant at the Oral Mucosa Dysplasia Clinic of the Dublin Dental University Hospital, the samples being taken from clinically evident, histologically-proven, and dysplastic oral lesions. All subjects were first evaluated by the clinician for the presence of histopathologically proven dysplasia and evident lesion. The brush-biopsy was a convenience sample of the patients with the lesion taken when the clinician was present in the Dysplastic Clinic. No adverse events were reported while collecting the brush-biopsy samples. Minor bleeding was reported in one case.

Histopathological assessment for the same patients from whom brush biopsy cytological samples were collected was performed. The biopsies were carried out by clinicians in the Oral Mucosa Dysplasia Clinic of the Dublin Dental University Hospital and were submitted to the histopathological laboratory in St. James' Hospital, Dublin, where they were reported by an oral maxillofacial pathologist using the current WHO 2017 guidelines for classification of oral epithelial dysplasia [27]. In the current study, the reference test used is a histopathological assessment of potentially malignant lesion samples. The sensitivity and specificity of the index test, that is Raman micro-spectroscopy, have been calculated to validate the classification models using PLS-DA. The details for the patients are given in Table 1 and the flowchart of participants included in the study is given in Figure 1.

The standardisation of the protocol, including collection tool, sample preparation, Raman data acquisition and processing for Raman micro-spectroscopic analysis of healthy donors has been reported earlier [26]. Standardisation of the protocol for sample collection mainly involves developing a method that ensures an adequate cell number, including cells from the basal layer on the slide to screen. To ensure proper sampling without bleeding, the collection site was vigorously rubbed (25 times) with the endocervical brush, using moderate pressure. This results in a good number of cells (typically 2500-6000 cells) on the slide for screening. Collected samples were placed into ThinPrep vials and sample slides were prepared using the ThinPrep2000 processor forming a monolayer of cells on glass slides for analysis. The standardised protocol was used to collect and process patient samples and healthy donor samples.

Collected samples were placed into ThinPrep vials and sample slides were prepared using the ThinPrep2000 processor forming a monolayer of cells on glass slides for analysis. The standardisation protocol was used to collect and process patient samples and healthy donor samples.

\section{2 | Raman spectral acquisition}

The protocol for spectral acquisition has been previously reported for ThinPrep cervical samples [19, 20]. An XploRA confocal Raman instrument (HORIBA JobinYvon) was used for spectral acquisition. Manual calibration of the grating was done at $520.7 \mathrm{~cm}^{-1}$ Raman band of crystalline silicon. Dark current measurement and recording of the substrate and optics signal was also 
T A B LE 1 Detailed information about the patients recruited for the study

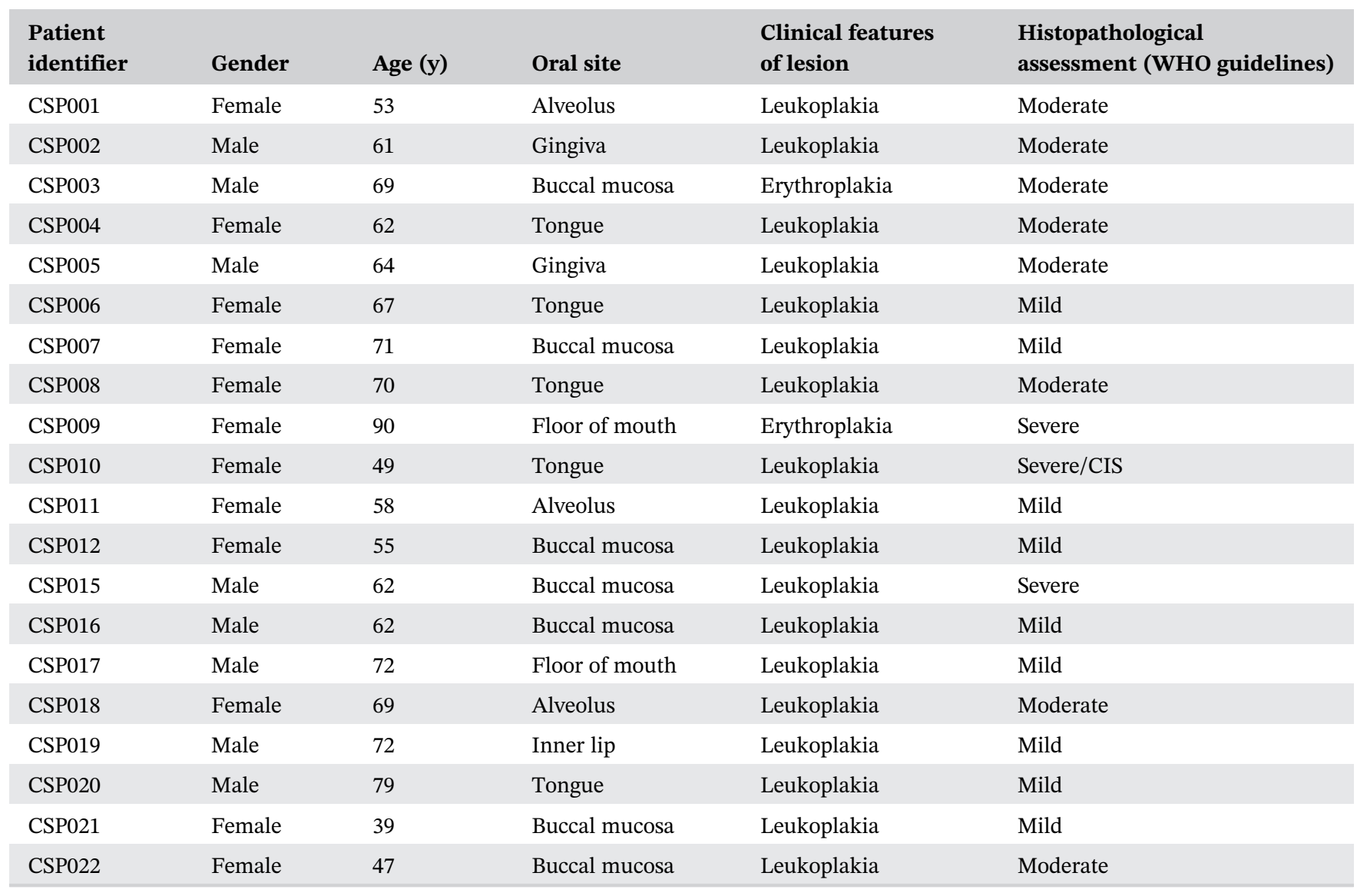

performed, for data correction. As a source, a $532 \mathrm{~nm}$ diode laser was focused by a 100X objective (MPlanN, Olympus, NA $=0.9)$ onto the sample $(\sim 12 \mathrm{~mW}$ at the focus) and the resultant Raman signals were detected using a spectrograph with a $1200 \mathrm{~g} \mathrm{~mm}^{-1}$ grating coupled with a CCD. Raman spectra were acquired in the 400 to $1800 \mathrm{~cm}^{-1}$ region with an integration time of 30 seconds per spectrum and averaged over two accumulations. As it is not known whether the nucleus or cytoplasm have greater diagnostic potential, spectra were acquired from the centre of the nucleus and at random from the cytoplasmic regions of the cells. Approximately 10 to 25 cells were recorded per slide, depending on the quality of the sample. Although a small voxel $(\sim 1 \mu \mathrm{m})$ is sampled each time, randomly sampling over multiple (10-25) cells means that the distribution of samplings effectively represents the variability across the cellular region [28-30].

\section{3 | Raman spectral processing and analysis}

All the data processing and analyses were carried out using Matlab (Mathworks, USA), PLS-Toolbox (Eigenvector Research, Inc) using in-house algorithms.
The glass spectral interference with the biological Raman signals was removed using the extended multiplicative signal correction (EMSC), as described by Kerr et al [31]. The EMSC algorithm also includes a polynomial term, and order $n=5$, was used to correct the baseline of the Raman spectral dataset. After glass correction, the datasets for nucleus and cytoplasm were subjected to smoothing (Savitzky-Golay, order $=5$; window $=13$ ), outlier removal using Grubb test [32] and vector normalisation.

Subsequently, pre-processed spectra of nucleus and cytoplasm were subjected to PLS-DA. PLS-DA was used to build models in order to train the dataset. PLS-DA is a supervised form of multivariate analysis which works as a linear classifier that aims to maximise the variance between groups and minimise the variance within groups. The loadings of the discriminate, that is latent variables (LVs) can be plotted to give more information on the source of the variance [33].

Cross validation of PLS-DA classification is carried out in order to avoid over or under-fitting the model due to inappropriate selection of the components used, and secondly to determine the prediction error of the model. In leave one patient out cross validation, (LOPOCV) or leave one spectrum out cross validation (LOOCV), observations of one patient/spectrum are excluded, one at a 
F I G U RE 1 Flowchart of participants. Index test = Raman micro-spectroscopic test; reference test $=$ histopathological assessment

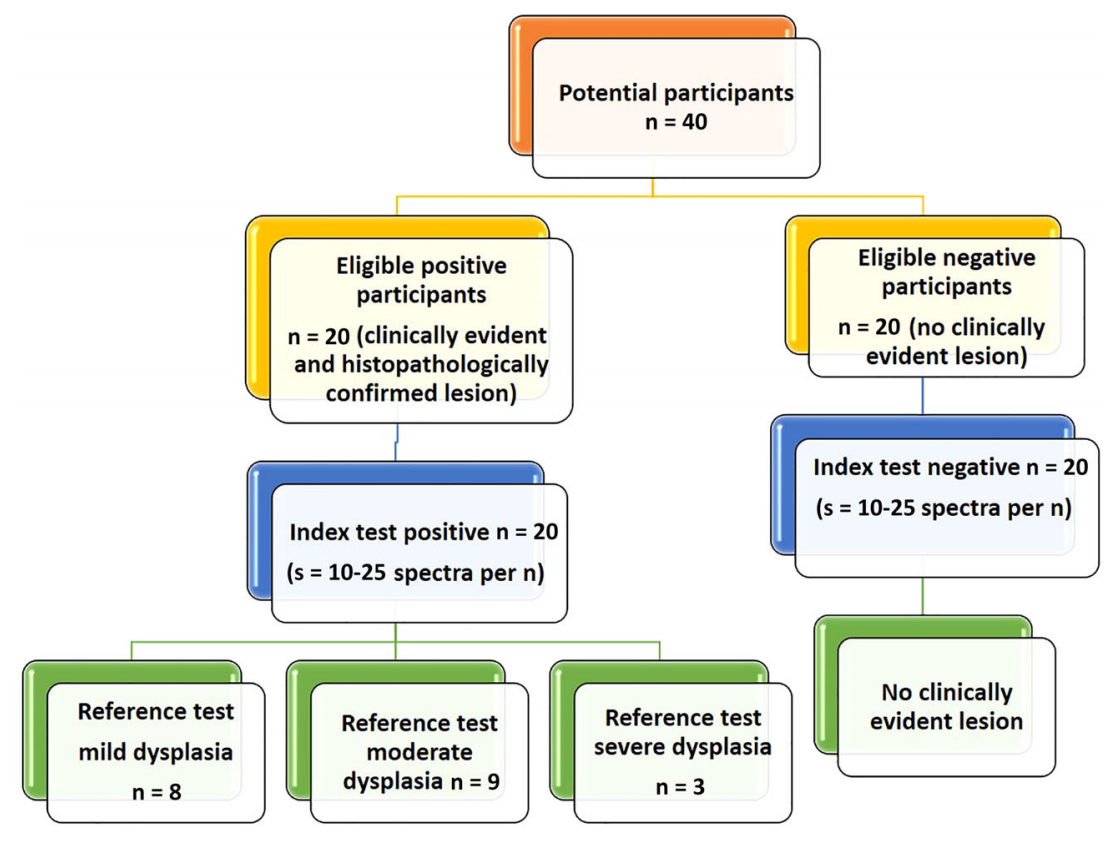

time, from the training set and the resulting model is evaluated by using the left out observations as tests. The procedure is repeated until all patients/spectra have been left out once and the average performance across all interactions is considered the performance of the classification model [34]. The one-way analysis of variance (ANOVA) was employed for calculating the significance of the outcomes for PLS-DA scores of healthy donors and patient samples in terms of $P$-values [35, 36].

\section{3 | RESULTS AND DISCUSSION}

In the present study, brush biopsy cytological samples were collected from four sites in healthy donors $(n=20)$ and from patients $(n=20)$ with identified leukoplakia, erythroplakia or erythroleukoplakia, showing mild, moderate or severe dysplasia on histological analysis.

Representative Papanicolaou (Pap) stained oral cytological samples can be seen in Figure 2. The samples obtained from mild and moderately dysplastic lesions mainly consisted of morphologically normal appearing cells, as shown in Figure 2A,B. However, increased numbers of abnormal cells, along with normal appearing cells, were observed in the samples from severely dysplastic lesions. In Figure 1C, the red arrow highlights an example of an abnormal cell in a severely dysplastic lesion. Raman spectra were acquired randomly from the samples, of morphologically normal and abnormal cells, from lesions reported as mild, moderate and severe dysplasia.

The mean spectra of cytoplasmic and nuclear regions of the cells from patients with potentially malignant lesions and healthy donors show similar spectral profiles, as can be seen in Figure 3A,B. The shading of the spectra indicates the SD. To better elucidate the subtle spectral differences between the healthy donor and patient samples, the difference spectra were also computed for both cytoplasmic and nuclear datasets (Figure 3A,B), subtracting the mean spectrum of patient samples from the mean spectrum of healthy donor samples. In Figure 3A, $\mathrm{B}$, all positive bands correspond to healthy donors, while the negative bands were from the patient samples. Table 2 illustrates the major Raman band assignments [37-42] used in the present study of healthy donors and patients with potentially malignant lesions. In the case of cytoplasm, protein $(636,845,997,1237,1361,1597$ and $\left.1645 \mathrm{~cm}^{-1}\right)$ and lipid $\left(1437 \mathrm{~cm}^{-1}\right)$ derived bands were observed to be prominent for healthy donor samples, whereas, for patient samples, nucleic acid (721, 780, $1180 \mathrm{~cm}^{-1}$ ) and lipid (1060, 1135, 1300, 1417 and $1745 \mathrm{~cm}^{-1}$ ) bands were observed. In the nuclear dataset, prominent protein $(636,842,994,1234,1361,1482,1575$, $\left.1665 \mathrm{~cm}^{-1}\right)$ and nucleic acid $\left(780,1482,1575 \mathrm{~cm}^{-1}\right)$ bands were observed for healthy donors, lipid $(712,938$, 1060, 1300, $1745 \mathrm{~cm}^{-1}$ ) and protein (938, 1460, $1648 \mathrm{~cm}^{-1}$ ) bands for patient samples. A predictive model based on the Raman micro-spectroscopic analysis was developed using PLS-DA with LOPOCV. The score plots of PLS-DA indicated that both LV1 and LV2 contribute to the differentiation of patient and healthy volunteer samples for both cytoplasmic (Supporting Information Figure S1) and nuclear (Figure 4A) datasets.

In the case of PLS-DA of the nuclear dataset (Figure 4B), negative bands of the LV1 loading, corresponding to samples from potentially malignant 
(A) Mild

\section{(B) Moderate}
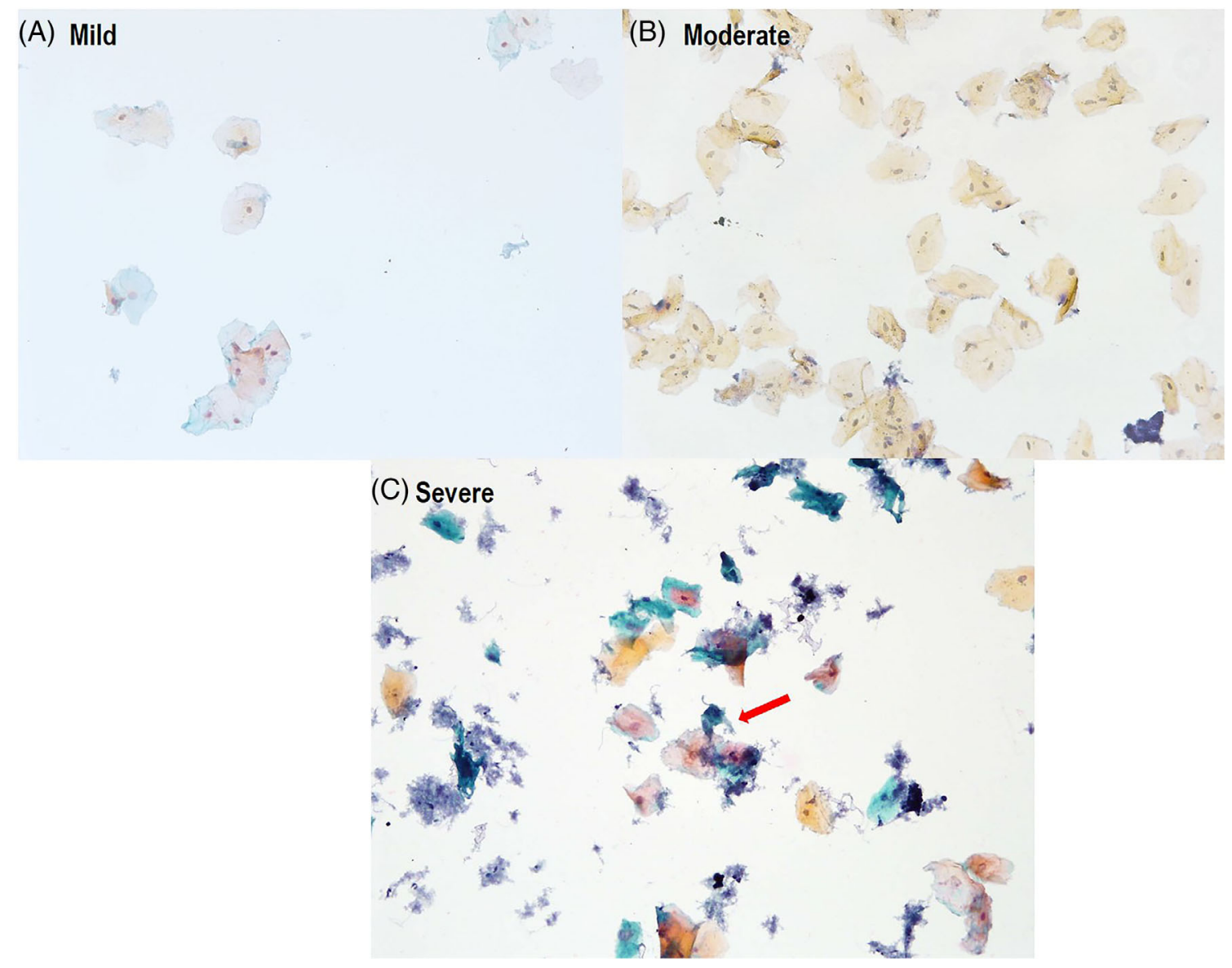

F I G U RE 2 Representative Pap stained oral cytological sample (20x) from lesions reported as A, mild; B, moderate and C, severe dysplasia. In $\mathrm{C}$, the red arrow highlights an example of an abnormal cell
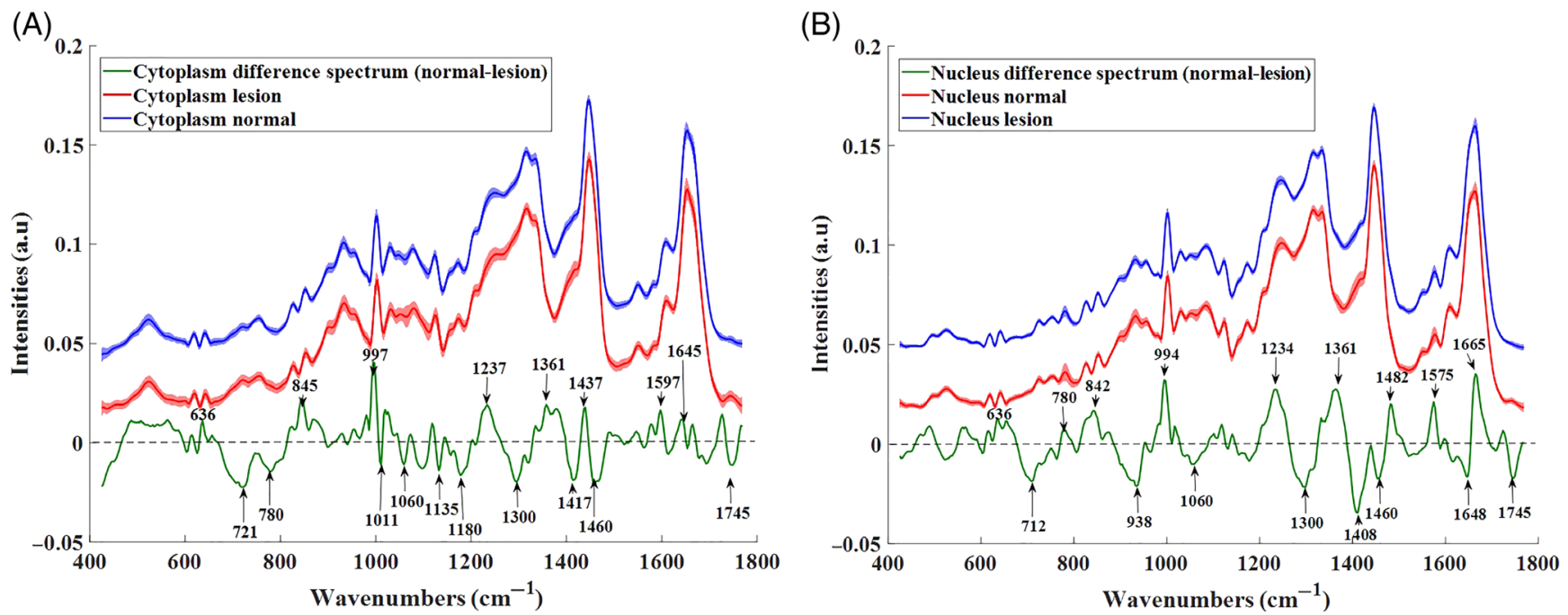

F I G U RE 3 Mean and difference spectra of healthy donors (normal) and patients (lesions) for A, cytoplasmic and B, nuclear dataset. Shading denotes SD. Note: the $y$-scale of the difference spectrum is different to that of the mean spectra

lesions, were of lipids (602, 705. 1132, 1155, 1300, $\left.1750 \mathrm{~cm}^{-1}\right)$, whereas prominent nucleic acid bands (726, 780, 828, 1094, 1369, 1485, $1572 \mathrm{~cm}^{-1}$ ) were observed in the cells from healthy donors on the positive bands of LV1 loading. The loading of LV2 showed a similar prominence of lipidic $\left(1065,1300,1745 \mathrm{~cm}^{-1}\right)$ features 
T A B L E 2 Major Raman band positions and assignments [38-43] in oral cytology for healthy donors and patients

\begin{tabular}{lc} 
Biomolecule & Raman bands $\left(\mathbf{c m}^{-\mathbf{1}}\right)$ \\
\hline Proteins & $521,534,571,628-662,830-835,859,890,921,935,952,963,994-1008,1028,1040,1163,1177$, \\
& $1197,1200,1228,1234,1259,1273,1361,1454,1460,1470,1532,1544,1600,1648,1655-80$ \\
\hline Lipids & $602,705,712,715,1060-1065,1130-1135,1146,1155,1300,1392,1417,1437,1465,1642,1673,1730-1760$ \\
\hline Nucleic acids & $720-730,780-785,1090-1095,1180,1296,1346,1361,1369,1482-1485,1572-1578$ \\
\hline
\end{tabular}

(A)

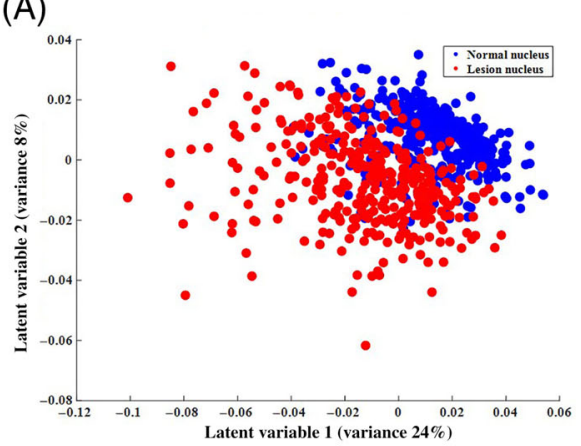

(B)

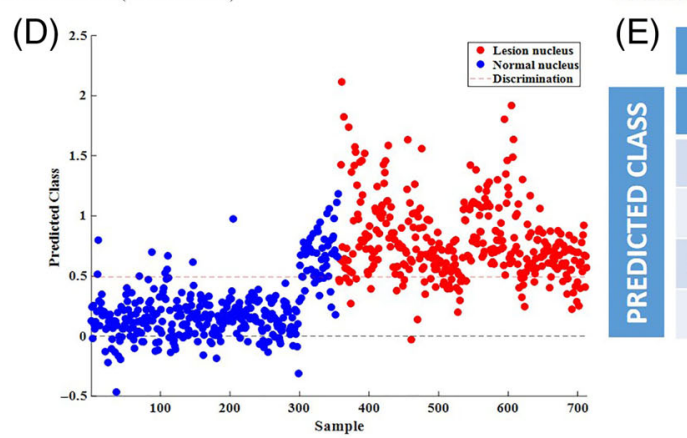

$(\mathrm{E})$

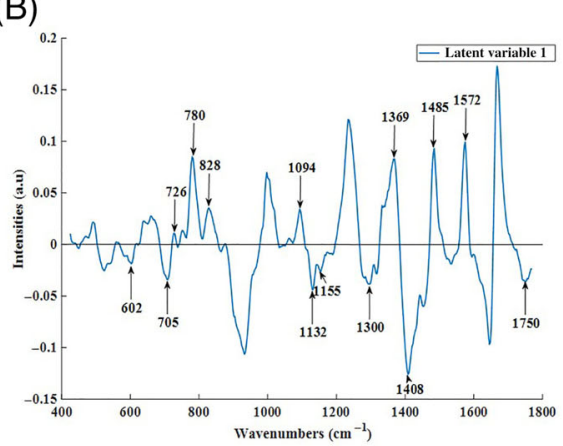

(C)

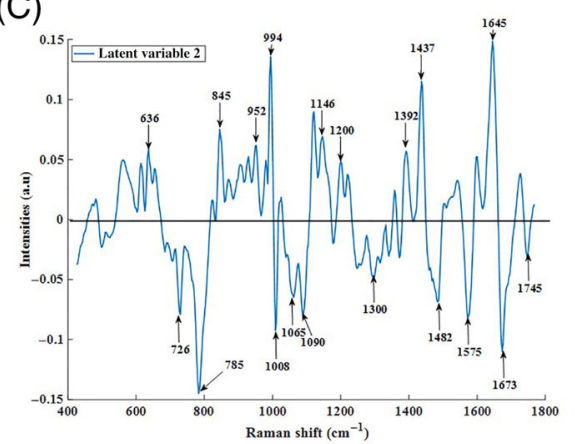

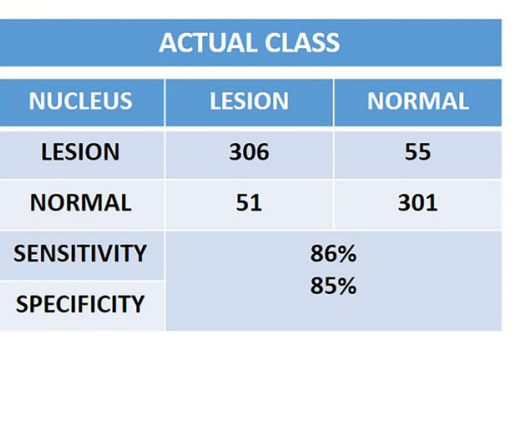

F I G U R E 4 PLS-DA predictive model for nucleus (normal vs lesion). A, Score plot; B, latent variable 1; C, latent variable 2; D, predictive model and E, confusion matrix

for the patient samples. In the case of patient samples, bands located at 1060, 1300, and $1750 \mathrm{~cm}^{-1}$ in LV1 and LV2 are consistent with the observations of the difference spectrum of Figure 3B, whereas for healthy donor samples, the $1437 \mathrm{~cm}^{-1}$ band in LV2 is also prominent in the difference spectrum. The predicted probability of the cellular spectroscopic measurement being assigned as normal or dysplastic is shown in the PLS-DA model with LOPOCV (Figure 4C). The confusion matrix of the model indicates $86 \%$ sensitivity (95\% CI $82 \%-89 \%$ ) and $85 \%$ specificity (95\% CI 80\%-88\%) for the nuclear dataset (Figure 4D). Similarly, in the case of cytoplasm, potentially malignant lesion samples were found to be relatively rich in lipids (LV1; 602, 1130, 1465, $1750 \mathrm{~cm}^{-1}$ and LV2 1065, 1437, $1740 \mathrm{~cm}^{-1}$ ), as shown in Supporting Information Figure S1b. Yet again, in the case of patient samples, bands located at 1060, 1300, and $1750 \mathrm{~cm}^{-1}$ of LV1 and LV2 are consistent with the observations of the difference spectrum, whereas unlike the nucleus, in the case of the cytoplasm, the $1437 \mathrm{~cm}^{-1}$ band is present in patient samples of LV2 instead of healthy donors. The confusion matrix (Supporting Information Figure S1d) of the model indicates $94 \%$ sensitivity (95\% CI 91\%-96\%) and $85 \%$ specificity (95\% CI 80\%-88\%).

Typically, a cytopathological assessment is based on morphological differences between healthy and patient samples. In certain cases, such as mild dysplasia, cellular changes occur near the basal layer, deep within the epithelium, which makes it difficult to harvest the abnormal cells in typical cytological samples. This can lead to false negative results, which reduce the sensitivity to as low as 69\% [43]. In this pilot study, Raman spectra were acquired from morphologically normal and abnormal cells from patients with mild, moderate and severely dysplastic lesions, and discriminative sensitivity of $94 \%$ and $86 \%$ was achieved for both the cytoplasmic and the nuclear datasets respectively. Hence, successful classification models for patient samples and healthy donor samples could be developed. 
The prominence of lipidic features in both the mean difference spectra and the discriminating LVs suggests altered lipid metabolisation pathways in the patient samples compared to the healthy donor samples and it may be explained by reprogramming of lipids during disease progression to fulfil the increased energy requirements [44] Notably, however, for both the cytoplasmic and nuclear regions, the lipid ester band at $1745 \mathrm{~cm}^{-1}$ is not so prominent in the multivariate discriminant analysis as it appears in the difference of the mean spectra in Figure 3. It is well known that, with increasing severity of dysplasia or cancer, the lipidic content of cells increases in the cytoplasm as well as in the nucleus [44, 45]. This can be attributed to the fact that, during cell division, cells need energy, and this requirement is fulfilled by upregulating lipid metabolism within the cell [44, 45]. In our study, increased lipidic content is the dominant differentiating factor of potentially malignant lesion samples for both the cytoplasmic and nuclear datasets. Furthermore, the results also suggest that morphologically normal cells of potentially malignant lesions are not biochemically normal and have the potential to transform into abnormal cells. Raman microspectroscopy can detect these subtle changes which are not as yet manifest morphologically. In this way, it can compensate for the fact that cytopathological sampling may not harvest dysplastic cells at or near the basal layer.

Subsequently, oral site as a confounding factor was tested in order to assess its influence on the PLS-DA predictive model. To facilitate the understanding of oral site as a confounding factor, it has been depicted in the predictive model for both cytoplasm and nuclear datasets respectively, as shown in Supporting Information Figure S2 and Figure 5. The four different sites; gingiva, alveolar, buccal mucosa and ventral surface of the tongue were considered, with the aim of understanding the influence of different anatomical sites on the analysis protocol for healthy donors and patients respectively for each site (Supporting Information Figure S2b and Figure 5B). It was observed that gingival and alveolar spectra from healthy donors were misclassified with patient samples for both cytoplasmic as well as the nuclear datasets (Supporting Information Figure S2b and Figure 5B). Even though, the $P$ values were found to be less than .00001 for the scores of PLS-DA (cytoplasm and nucleus) for healthy donors and patient samples using one-way ANOVA, due to misclassification of gingival and alveolar spectra with patient samples the overall efficiency of the model has been reduced which can be seen in Supporting Information Figure S2 and Figure 5.

In order to account for the influence of different sites on the predictive model, gingiva/alveolus mucosa samples $(n=3)$ were removed from the overall dataset. Using only the remaining buccal mucosa/tongues samples, PLS-DA classification models for healthy donors and patients were developed which are shown in Supporting Information Figure S3 for the cytoplasmic dataset and Figure 6 for the nuclear dataset. In both, it is possible to distinguish healthy donors from patient samples according to LV 1 and LV 2, which can be seen in the scores plot of PLS-DA (Supporting Information Figure S3a and 6a). The LV1 and LV2 for cytoplasm as well as nucleus respectively were similar to those in Supporting Information Figure S1 and Figure 4. In the case of PLS-DA of the nuclear dataset (Figure 6B), negative bands at of LV1, corresponding to potentially malignant lesions, were of lipids [39, 40], whereas prominent DNA bands were observed in the cells from healthy
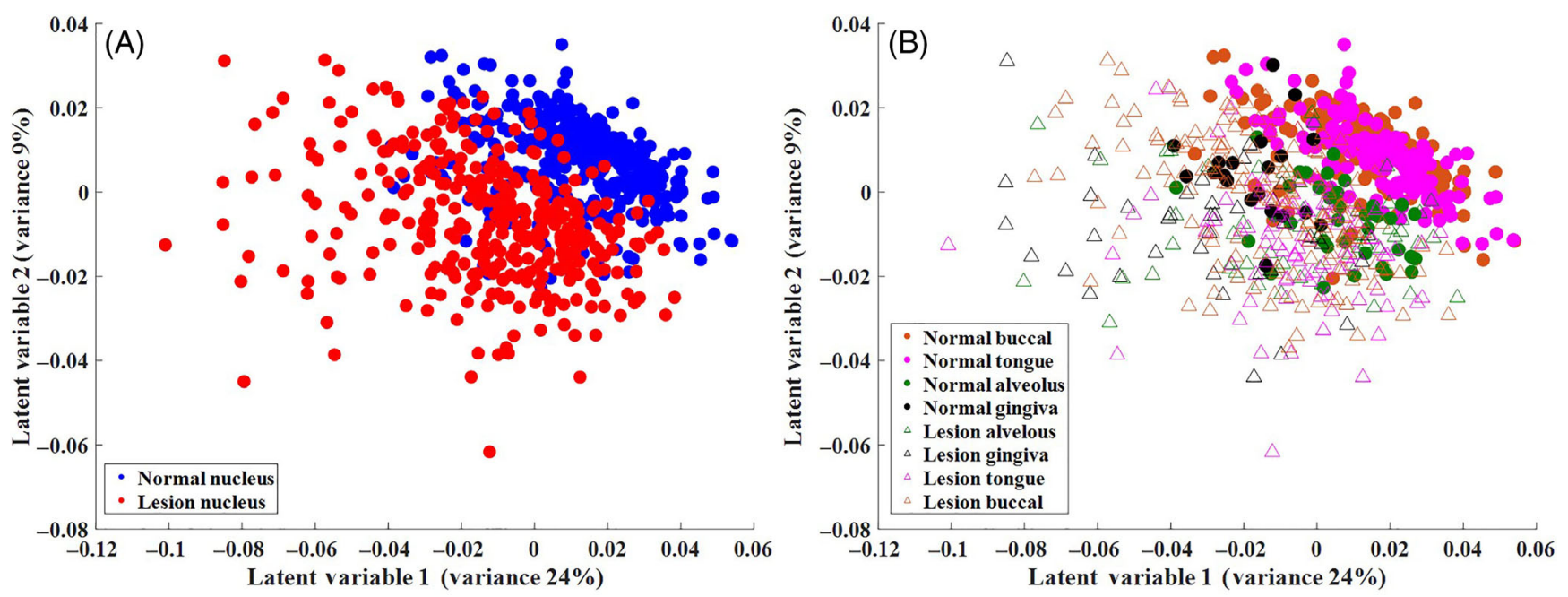

F I G U R E 5 A, PLS-DA score plot for nuclear spectra from healthy donors (normal) vs patients (lesions). B, Depicting anatomical sites. (One-way ANOVA, $P<.00001$ for both LV1 and LV2) 
donors as the positive bands [39, 40]. The lipid bands were also observed in LV2 of negative bands corresponding to the patient samples. The confusion matrix of the model indicated $97 \%$ sensitivity/specificity for the cytoplasmic dataset (Supporting Information Figure S3e), whereas $94 \%$ sensitivity and $95 \%$ specificity was achieved in the case of the nuclear dataset (Figure 6E). For the cytoplasm, negative bands of LV1 loading corresponding to potentially malignant lesion samples were found to be rich in lipids and DNA bands $[39,40]$, as shown in Supporting Information Figure S3b. It was also observed that the positive bands of LV1, corresponding to healthy donors, were predominantly of proteins $[39,40]$, as can be seen in Supporting Information Figure S3b. Similarly, lipids bands were observed in patient samples on LV2. This has suggested that the model can be effectively improved by developing two separate models for gingiva/alveolus and buccal mucosa/tongue samples. Therefore, it has been concluded that, for the future studies, two separate models will be developed based on difference in oral sites.

After confirming oral sites as a confounding factor, the influence of various other potential confounding factors such as gender, age, smoking and alcohol consumption were assessed for their influence on the buccal/ tongue PLS-DA classification model for healthy donors and patients. Firstly, gender is illustrated in the scores plot of the PLS-DA model for cytoplasm and nucleus
(Supporting Information Figure S4a and Figure 7A) using six females and nine males for healthy donors and nine females and six males for patient samples. Secondly, age profile is shown in the classification model of the potentially malignant lesion samples $(50+$ years $(\mathrm{n}=13)$ and under 50 years $(\mathrm{n}=2))$ and healthy donors $(20-30$ years $(\mathrm{n}=10), 30-50$ years $(\mathrm{n}=4), 50+$ years $(\mathrm{n}=1))$ in Supporting Information Figure S4b and Figure 7B. The third potential confounding factor that was considered is smoking, which is depicted in the scores plot of the PLSDA scatter plot (Supporting Information Figure S4c and Figure $7 C$ ) for patient samples (smokers $(\mathrm{n}=3)$, exsmokers $(\mathrm{n}=6)$, non-smokers $(\mathrm{n}=6))$ and healthy (smokers $(n=4)$, non-smokers $(n=11)$ ). Finally, alcohol consumption was examined as the fourth potential confounding factor, shown in Supporting Information Figure S4d and Figure 7D, using patient samples (no alcohol $(\mathrm{n}=5)$ and alcohol consumption $(\mathrm{n}=10)$ ) and healthy donor samples (no alcohol $(\mathrm{n}=3)$ and alcohol consumption $(\mathrm{n}=12)$ ). From the scores plot of the PLS-DA (Supporting Information Figure S4 and Figure 7) of patients with potentially malignant lesions and healthy donors, it is clear that, irrespective of gender, age, smoking and alcohol consumption, it is possible to distinguish healthy donors from patient samples according to LV 1 for the cytoplasmic as well as the nuclear dataset. A one-way ANOVA test indicated $P$ values lower than .0001 for the scores of healthy donors and patient samples
(A)

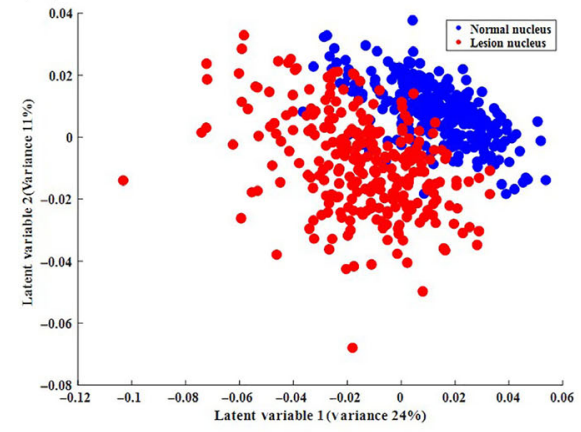

(B)

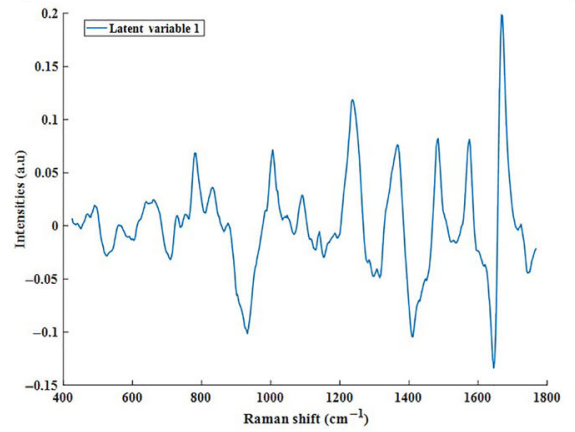

(C)

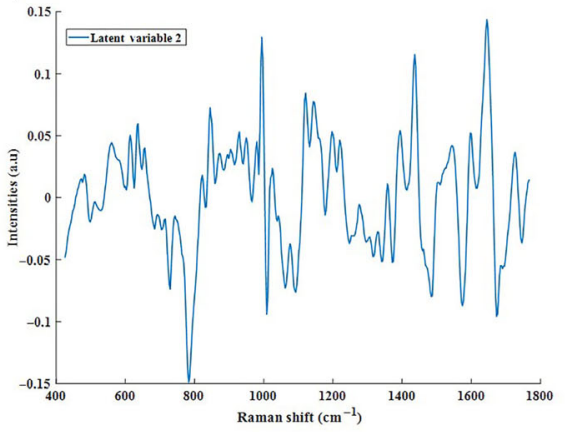

(D)

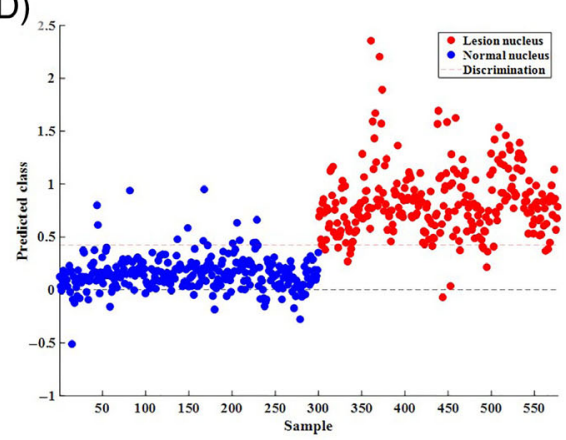

\begin{tabular}{|c|c|c|c|}
\hline (E) & \multicolumn{3}{|c|}{ ACTUAL CLASS } \\
\hline \multirow{5}{*}{$\begin{array}{l}\frac{y}{5} \\
\frac{0}{0} \\
\frac{0}{2} \\
\frac{0}{0}\end{array}$} & NUCLEUS & LESION & NORMAL \\
\hline & LESION & 260 & 13 \\
\hline & NORMAL & 17 & 287 \\
\hline & SENSITIVITY & \multirow{2}{*}{\multicolumn{2}{|c|}{$\begin{array}{l}94 \% \\
95 \%\end{array}$}} \\
\hline & SPECIFICITY & & \\
\hline
\end{tabular}

FI G U RE 6 PLS-DA predictive model for nucleus spectra from buccal mucosa/tongue (normal vs lesion). A, Score plot; B, latent variable $1 ; \mathrm{C}$, latent variable $2 ; \mathrm{D}$, predictive model and $\mathrm{E}$, confusion matrix 

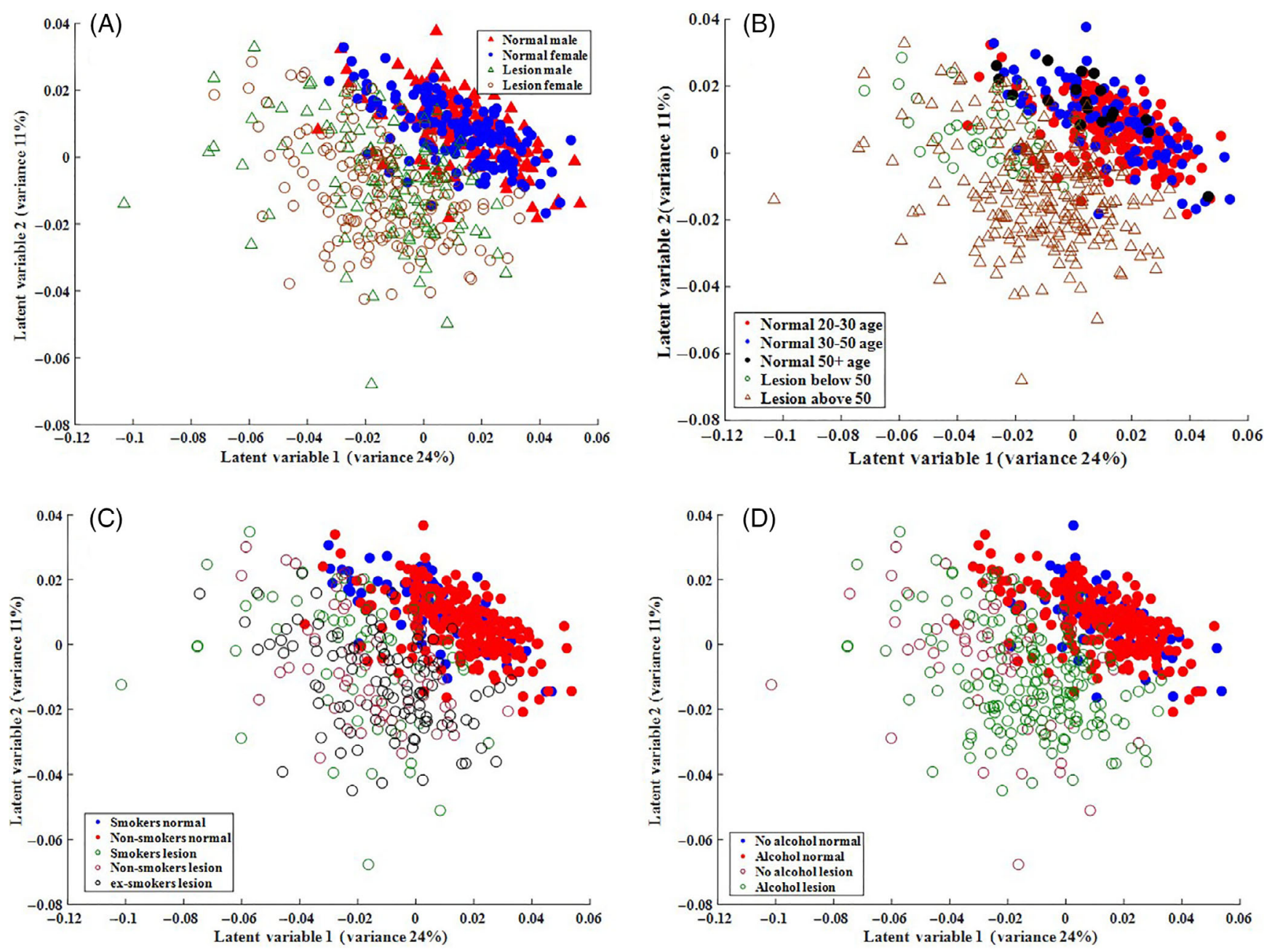

FI G URE 7 PLS-DA predictive model for nucleus spectra for healthy donors (normal) vs patient samples (lesion). A, Score plot gender; $\mathrm{B}$, score plot age; $\mathrm{C}$, score plot smoking and $\mathrm{D}$, score plot alcohol consumption. (One-way ANOVA, $P<.00001$ )

(in Supporting Information Figure S4 and Figure 7). Since the overall sensitivity of the model has been improved after the removal of the gingival and alveolar samples, it was concluded that gender, age, smoking or alcohol consumption habits do not confound the Ramanbased classification model.

The result suggests that any gender, age, smoking and alcohol consumption dependent morphological differences were not reflected at the molecular level and were not manifest in the Raman spectral profiles. The samples for confounding factors such as gender, smoking and alcohol consumption were approximately matched. However, the age distribution was, to a certain extent, biased towards younger donors. Nevertheless, this age distribution was not seen to be a factor in the spectral profile of normal samples. Thus, it can be concluded that the healthy donor or patient datasets are not influenced by gender, age, smoking or alcohol consumption, while using Raman micro-spectroscopy as a diagnostic technique.

\section{4 | CONCLUSION}

Raman micro-spectroscopy was found to be able to successfully discriminate oral cytology samples from patients with potentially malignant lesions from those of healthy donors with $94 \%$ and $86 \%$ sensitivity, based on analysis of both cytoplasmic and nuclear regions. Morphologically normal cells within potentially malignant lesions were identified as abnormal due to the biochemical differences manifest in their Raman spectroscopic profiles. Thus, Raman micro-spectroscopy can detect subtle changes in cells that could be falsely diagnosed as normal based on morphology alone. Hence, this study has shown that the limitations associated with the cytopathological assessment of oral dysplastic lesions could be successfully overcome by Raman micro-spectroscopy, which identifies biochemical differences in the morphologically normal cells within these dysplastic oral lesions.

Furthermore, even though for the present study the spectra were acquired from the morphologically normal 
cells of potentially malignant lesion samples, all the patient samples which were included in the study were from histopathologically confirmed dysplastic lesion samples. Thus, through our study, the dysplastic patient samples could be accurately differentiated from nondysplastic healthy donor samples using Raman microspectroscopy and brush biopsy samples. This, in turn, has implications in clinical practice to reduce the need for multiple biopsies for screening the presence of disease. In future, a study will be carried out for histologically classified dysplastic lesions versus non-dysplastic lesions.

It was, however, observed that the Raman spectral profiles of cells from gingiva/alveolus sites were distinctively different from those from the buccal mucosa/ventral surface of the tongue in healthy donors. Moreover, it was also observed that Raman spectra from gingiva/alveolus mucosa samples from healthy volunteers were misclassified with potentially malignant lesion samples. Thus, it was concluded that oral site is a confounding factor for diagnostic applications and can have an impact on classification models. Anatomical selectivity can clearly be controlled in the clinical sampling protocol, and thus is not a significant impediment for the further development of the label free analysis technique for screening of potentially malignant lesions.

Furthermore, this study has indicated that although morphological changes have been reported due to differences in gender, age, smoking, and alcohol consumption in the cells of healthy donors, these changes are not manifested at the molecular level to be detected by Raman micro-spectroscopy. Therefore, it has been concluded that gender, age, smoking and alcohol have no influence on developing classification models for healthy donors and patients using Raman micro-spectroscopy.

At present, the adoption of Raman spectroscopy as a diagnostic method to detect degrees of dysplasia in oral mucosal cells is at the "proof of concept" stage, but results are very encouraging. In the future, this study will be carried out with a larger number of samples and it should be possible to develop the technique, such that it can be used in vivo, with real-time data analysis. The technique represents a potentially powerful tool to assist in directing the clinician to the most appropriate biopsy site for extensive leukoplakic lesions (Raman-directed biopsy). It could also be an intra-operative surgical aid in locating the excision margin [46] of tumours, so all dysplastic tissue is removed where clinically feasible.

\section{ACKNOWLEDGMENT}

This research was funded by the Technological University Dublin, Fiosraigh Dean of Graduate Studies Award and Science Foundation Ireland (12/IP/1494).

\section{CONFLICT OF INTEREST}

The authors declare no conflicts of interest.

\section{ORCID}

Isha Behl (D) https://orcid.org/0000-0003-0676-8988

Hugh J. Byrne (1) https://orcid.org/0000-0002-1735-8610

Fiona M. Lyng (1) https://orcid.org/0000-0002-9876-963X

\section{REFERENCES}

[1] GLOBOCAN: Estimated Cancer Incidence, Mortality and Prevalence Worldwide in 2018, International Agency for Research on Cancer, 2018, (online), http://globocan.iarc.fr/Pages/fact_ sheets_population.aspx (accessed: March 2020).

[2] C. F. Poh, C. E. MacAulay, D. M. Laronde, P. M. Williams, L. Zhang, M. P. Rosin, Periodontol 2000 2011, 57(1), 73.

[3] B. W. Neville, T. a Day, Ca-Cancer J. Clin. 2002, 52(4), 195.

[4] P. V. Centelles, J. M. Seoane-romero, I. Gómez, P. Diz-dios, N. S. De Melo, J. Seoane, Timing of Oral Cancer Diagnosis: Implications for Prognosis and Survival, Oral Cancer, Kalu U. E.Ogbureke, IntechOpen, 2012. https://doi.org/10.5772/30591.

[5] I. J. C. Schneider, M. E. Flores, D. A. Nickel, L. G. T. Martins, J. Traebert, Rev. Bras. Epidemiol. 2014, 17, 680.

[6] A. Trakroo, M. K. Sunil, A. Trivedi, R. Garg, A. Kulkarni, S. Arora, J. Int. Oral Health 2015, 7(3), 33.

[7] N. V. Babshet M, K. Nandimath, S. Pervatikar, J. Cytol. 2011, 28(4), 165.

[8] T. A. Winning, G. C. Townsend, Clin. Dermatol. 2000, 18 (5), 499.

[9] C. A. Squier, M. J. Kremer, J. Natl. Cancer Inst. Monogr. 2001, 52242(29), 7.

[10] V. Kumar, N. Fausto, A. Abbas, Robbins and Cotran Pathologic Basis of Disease, 7th ed., Elsevier, Amsterdam, 2005.

[11] C. Scully, J. V. Bagan, C. Hopper, Am. J. Dent. 2008, 21(4), 200.

[12] K. Papamarkakis, B. Bird, J. M. Schubert, M. Miljković, R. Wein, K. Bedrossian, N. Laver, M. Diem, Lab. Invest. 2010, 90 (4), 589.

[13] V. G. Patel, K. S. PV, V. Kumar, J. Cytol. 2011, 28(2), 66.

[14] D. Wynford-Thomas, J. Pathol. 1999, 187(1), 100.

[15] A. V. O.-P. Alejandra Isabel Orellana-Bustos, I. L. EspinozaSantander, M. E. Franco-Martínez, N. Lobos-James-Freyre, Med. Oral 2004, 9(3), 197.

[16] N. K. Proia, G. M. Paszkiewicz, M. A. S. Nasca, G. E. Franke, J. L. Pauly, Cancer Epidemiol. Biomarkers Prev. 2006, 15, 1061.

[17] P. A. Wu, C. H. Loh, L. L. Hsieh, T. Y. Liu, C. J. Chen, S. H. Liou, Mutat. Res., Genet. Toxicol. Environ. Mutagen. 2004, 562 (1-2), 27.

[18] M. R. Khairnar, U. Wadgave, S. M. Khairnar, J. Alcohol. Drug Depend. 2017, 5(3), 3.

[19] S. Regina, D. A. Reis, M. Gustavo, S. Andrade, M. Sadigursky, Development 2006, 20(2), 97.

[20] M. Diem, K. Papamarkakis, J. Schubert, B. Bird, M. J. Romeo, M. Miljković, Appl. Spectrosc. 2009, 63(11), 307.

[21] M. Diem, A. Mazur, K. Lenau, J. Schubert, B. Bird, M. Miljković, C. Krafft, J. Popp, J. Biophotonics 2013, 6 (11-12), 855.

[22] M. Miljković, B. Bird, K. Lenau, A. I. Mazur, M. Diem, Analyst 2013, 138, 3975. 
[23] A. Sahu, N. Shah, M. Mahimkar, M. Garud, S. Pagare, S. Nair, C. M. Krishna, in Proc. SPIE 8926, Photonic Therapeutics and Diagnostics X, 89262N, 2014.

[24] A. Sahu, P. Gera, V. Pai, A. Dubey, G. Tyagi, M. Waghmare, S. Pagare, M. Mahimkar, C. M. Krishna, J. Biomed. Opt. 2017, 22 (11), 1.

[25] A. Sahu, S. Tawde, V. Pai, P. Gera, P. Chaturvedi, C. M. Krishna, Anal. Methods 2015, 7, 7548.

[26] I. Behl, G. Calado, O. Ibrahim, A. Malkin, S. Flint, H. J. Byrne, F. M. Lyng, Anal. Methods 2017, 9(6), 937.

[27] A. K. El-Naggar, J. K. C. Chan, J. R. Grandis, T. Takata, P. J. Slootweg Eds., WHO Classification of Head and Neck Tumours, 4th ed., IARC, France, 2017.

[28] H. Nawaz, F. Bonnier, A. D. Meade, F. M. Lyng, H. J. Byrne, Analyst 2011, 136, 2450.

[29] F. Bonnier, H. J. Byrne, Analyst 2012, 137(2), 322.

[30] Z. Farhane, F. Bonnier, A. Casey, A. Maguire, L. O'Neill, H. J. Byrne, Analyst 2015, 140, 5908.

[31] L. T. Kerr, B. M. Hennelly, Chemom. Intel. Lab. Syst. 2016, 158, 61.

[32] M. Urvoy, F. Autrusseau, in Proc. 2nd ACM Work. Inf. Hiding Multimed. Secur., IH\&MMSec'14, 2014, pp. 49-60.

[33] R. G. Brereton, G. R. Lloyd, J. Chemometr. 2014, 28 (4), 213.

[34] R. Gautam, S. Vanga, F. Ariese, S. Umapathy, EPJ Tech. Instrum. 2015, 2(1), 8 .

[35] S. F. Sawyer, J. Man. Manip. Ther. 2009, 17(2), 27E.

[36] D. C. Montgomery, G. C. Runger, Appl. Stat. Probab. Eng. 7th ed., Wiley, Hoboken, NJ, 2018.

[37] C. Krafft, L. Neudert, T. Simat, R. Salzer, Spectrochim. Acta, Part A 2005, 61, 1529.

[38] M. Gniadecka, H. C. Wulf, N. N. Mortensen, O. F. Nielsen, D. H. Christensen, J. Raman Spectrosc. 1997, 28 (2-3), 125.
[39] Z. Movasaghi, S. Rehman, I. Rehman, Appl. Spectrosc. 2007, 42 (5), 493.

[40] H. Byrne, G. Sockalingum, N. Stone, Raman Microscopy: Complement or Competitor. in Biomedical Applications of Synchrotron Infrared Microspectroscopy, RSC Analytical Spectroscopy Series. 2011, p. 105.

[41] N. Stone, C. Kendall, J. Smith, P. Crow, H. Barr, Faraday Discuss. 2004, 126(1), 141.

[42] J. W. Chan, D. S. Taylor, T. Zwerdling, S. M. Lane, K. Ihara, T. Huser, Biophys. J. 2006, 90(2), 648.

[43] S. Kumar, N. Vezhavendhan, S. Priya, Int. J. Clin. Dent. Sci. 2011, 2(1), 293.

[44] S. Beloribi-Djefaflia, S. Vasseur, F. Guillaumond, Oncogenesis 2016, 5(1), e189.

[45] G. Cascianelli, M. Villani, M. Tosti, F. Marini, E. Bartoccini, M. V. Magni, E. Albi, Mol. Biol. Cell 2008, 19, 5289.

[46] F. L. Cals, T. C. B. Schut, J. A. Hardillo, R. J. B. de Jong, S. Koljenović, Lab. Invest. 2015, 95, 1186.

\section{SUPPORTING INFORMATION}

Additional supporting information may be found online in the Supporting Information section at the end of this article.

How to cite this article: Behl I, Calado G, Malkin A, et al. A pilot study for early detection of oral premalignant diseases using oral cytology and Raman micro-spectroscopy: Assessment of confounding factors. J. Biophotonics. 2020;13: e202000079. https://doi.org/10.1002/jbio.202000079 